\title{
PROXIMINALITY AND DIAMETRICALLY MAXIMAL SETS IN $C(K)$ *
}

\author{
J. P. MORENO ${ }^{\dagger}$
}

\begin{abstract}
We are concerned in this note with two optimization problems associated with diametrically maximal sets in spaces of continuous functions. Given an intersection of closed balls $C \subset C(K)$, we show that there is an optimal solution to: i) the problem of minimizing $\operatorname{dist}(C, E)$ where $E$ is any diametrically maximal set satisfying $\operatorname{diam} E=\operatorname{diam} C$, ii) the same problem removing the constraint diam $E=\operatorname{diam} C$. We provide a characterization of the uniqueness of solution in the first problem.
\end{abstract}

Key words. Diametrically maximal set, metric projection, proximinality, Chebyshev set, intersections of closed balls.

AMS subject classifications. 41A50, 41A52, 46B20, 46E15

1. Introduction. Spaces of continuous functions play a central role in several branches of analysis and have deep and far reaching applications in many other areas of mathematics. Some classical topics from convex geometry have been investigated in recent years in spaces of infinite dimension (see [2], [3], [23], [24] and [26]), with special attention to the case of $C(K)$ spaces [15], [16], as a contribution to a better understanding of its nonlinear and geometrical properties. This is the case of diametrically maximal sets [18], [19], a class of convex sets introduced by Meissner at the beginning of last century [14] as a counterpart to the older notion of constant width set [7], [14]. A closed, bounded and convex set $C$ in a normed space is said to be diametrically maximal if

$$
\operatorname{diam} C<\operatorname{diam}(C \cup\{y\})
$$

for every $y \notin C$ or, equivalently, if $C=\bigcap_{x \in C}(x+d B)$ where $B$ is the unit ball and $d=\operatorname{diam} C[9]$. This characterization, known as the spherical intersection property, shows that a diametrically maximal set is always an intersection of closed balls. The aim of this note is to answer the following question: how far from being diametrically maximal is an intersection of closed balls in $C(K)$ ?

Let us now make the above question more precise and see the connections with optimization and best covering problems. We will denote by $\mathcal{H}$ the family of all closed, convex and bounded sets in $C(K)$, endowed with the Hausdorff metric. The family of all diametrically maximal sets will be denoted by $\mathcal{D} \mathcal{M}$ and the family all intersections of closed balls by $\mathcal{M}$. It is clear, by the spherical intersection property, that $\mathcal{D} \mathcal{M} \subset \mathcal{M} \subset \mathcal{H}$.

Diametrically maximal sets are also called complete sets, and it is well known that every bounded set $C$ can be completed, in the sense that there is always a complete set $D$ containing $C$ such that $\operatorname{diam} C=\operatorname{diam} D[7]$. Such a set $D$ is called a completion of $C$. Given $C \in \mathcal{H}$, denote by $\gamma(C)$ the set of all completions of $C$. Some basic properties of the multivalued mapping $\gamma: \mathcal{H} \rightarrow 2^{\mathcal{H}}$ have been investigated in [16] (for connections between porosity and best approximation problems see also [25]) and

\footnotetext{
*Received September 1, 2009; accepted for publication April 30, 2010.

$\dagger$ Dpto. Matemáticas, Facultad de Ciencias, Universidad Autónoma de Madrid, Madrid 28049, Spain (josepedro.moreno@uam.es). Partially supported by the DGICYT project MTM 2009-07848.
} 
[17]. We are concerned in this paper with the following Problem $P$ : given $C \in \mathcal{M}$,

$$
\begin{aligned}
\text { Minimize } & \operatorname{dist}(C, E) \\
\text { subject to } & E \in \gamma(C)
\end{aligned}
$$

and the corresponding Problem $P^{\prime}$ obtained by replacing the above constraint by the weaker restriction $E \in \mathcal{D} \mathcal{M}$. Though spaces of continuous functions often present a variety of behaviors (actually they are often used as a source of counterexamples [13]), both problems have optimal solutions in every $C(K)$ space. We will provide a characterization of the uniqueness of the optimal solutions in Problem P.

Approximating or covering a convex set by a special family of convex bodies are classical subjects in convex geometry (see [1], [4], [27]), but it has been hardly explored in the context of hyperspaces. The study of optimization theory in metric spaces without linearity has received little attention as well (see [21], [29]), compared with the classical optimization theory in vector spaces. In this latter setting, proximinality (the existence of best approximations) is a main subject of research (see, for instance, [5], [6], [10], [11], [12], [20], Deutsch's book [8] and classical Singer's monograph [28]). One of our aims in this paper is considering this notion in a new context.

2. Notation and preliminaries. If $K$ is any compact Hausdorff space, the space $C(K)$ consists of all real-valued continuous functions $f: K \rightarrow \mathbb{R}$ under the sup norm $\|f\|=\max _{t \in K}|f(t)|$. As proved in [15], a closed, convex and bounded set $C \subset C(K)$ is a nonempty intersection of closed balls if and only if there are $f_{C}, g_{C}: K \rightarrow \mathbb{R}$ lower and upper semicontinuous functions, respectively, such that

$$
C=\left[f_{C}, g_{C}\right]=\left\{h \in C(K): f_{C}(t) \leq h(t) \leq g_{C}(t), t \in K\right\} .
$$

We will often use the fact that $f_{C}(t)=\inf \{h(t): h \in C\}$ when $t$ is a point of continuity for $f_{C}$ and, analogously, $g_{C}(t)=\sup \{h(t): h \in C\}$ if $t$ is a point of continuity for $g_{C}$ [15]. There is a useful characterization of $\gamma(C)$ by using the family $\Gamma(C)$ of those lower semi-continuous functions $\varphi: K \rightarrow \mathbb{R}$ such that

$$
g_{C}(t)-d_{C} \leq \varphi(t) \leq f_{C}(t)
$$

for every $t \in \mathcal{D}_{f_{C}} \cap \mathcal{D}_{g_{C}}$, where $\mathcal{D}_{f_{C}}$ and $\mathcal{D}_{g_{C}}$ stand for the points of continuity of $f_{C}$ and $g_{C}$, respectively. Indeed, it is known that

$$
\gamma(C)=\left\{\left[\varphi, \check{\varphi}+d_{C}\right]: \varphi \in \Gamma(C)\right\}
$$

where $\check{\varphi}$ denotes the upper semi-continuous envelope of $\varphi$, namely $\check{\varphi}(t)=$ $\limsup _{s \rightarrow t} \varphi(s)$, for every $t \in K[16]$. Besides, if $f^{\prime}, f^{\prime \prime} \in \Gamma(C)$ and $E^{\prime}=\left[f^{\prime}, \check{f}^{\prime}+d_{C}\right]$, $E^{\prime \prime}=\left[f^{\prime \prime}, f^{\prime \prime}+d_{C}\right]$ are their corresponding completions, then

$$
\operatorname{dist}\left(E^{\prime}, E^{\prime \prime}\right)=\sup _{t \in \mathcal{D}_{0}}\left|f^{\prime}(t)-f^{\prime \prime}(t)\right|
$$

where $\mathcal{D}_{0}$ is any dense set contained in $\mathcal{D}_{f^{\prime}} \cap \mathcal{D}_{f^{\prime \prime}}[16]$.

Together with the diameter, there is another constant relative to a set $C \in \mathcal{M}$ that will play a crucial role in this paper. Define

$$
\delta_{C}(t)=\sup \{|\varphi(t)-\psi(t)|, \varphi, \psi \in C\}
$$


for every $t \in K$ and denote by $\mathcal{D}_{\delta_{C}}$ the points of continuity of $\delta_{C}$, which is always a residual set since $\delta_{C}$ (as a supremum of continuous functions) is lower semicontinuous. Consider now

$$
\delta_{C}:=\inf \left\{\delta_{C}(t): t \in \mathcal{D}_{\delta_{C}}\right\}
$$

and denote by $d_{C}=\operatorname{diam} C$, namely the value obtained in (2.4) when the inf is replaced by a sup.

The metric projection $\pi(C)$ onto $\gamma(C)$ is defined as the family of all optimal completions, namely all $D \in \gamma(C)$ satisfying $\operatorname{dist}(C, D)=d(C, \gamma(C))$, where

$$
d(C, \gamma(C)):=\inf \{\operatorname{dist}(C, E): E \in \gamma(C)\} .
$$

Using the usual terminology from approximation theory, the set $\gamma(C)$ is said to be (i) proximinal $^{1}$ at $C$ when $\pi(C) \neq \emptyset$, (ii) Chebyshev at $C$ when $\pi(C)$ is a singleton.

3. When is $\gamma(C)$ proximinal at $C$ ? If $C$ is an intersection of balls, the numbers $d_{C}$ and $\delta_{C}$ are closely related to $d(C, \gamma(C)$ ), as formula (3.2) below highlights. Proposition 3.1 also shows that $\gamma(C)$ is proximinal at $C$. Said in other words, in $C(K)$ spaces, Problem $\mathrm{P}$ always has a solution.

Given $C=\left[f_{C}, g_{C}\right] \in \mathcal{M}$, we define a related set $E$ as follows. First, consider $\mathcal{D}:=\mathcal{D}_{f_{C}} \cap \mathcal{D}_{g_{C}}$ and set

$$
h_{0}(t)=1 / 2\left(g_{C}(t)+f_{C}(t)\right)
$$

for every $t \in \mathcal{D}$. Let $f_{0}(t)=h_{0}(t)-d_{C} / 2$ and $g_{0}(t)=h_{0}(t)+d_{C} / 2$, again for $t \in \mathcal{D}$. Define now $f$ and $g$ as the lower and upper semicontinuous extensions of $f_{0}$ and $g_{0}$, respectively:

$f(t)=\left\{\begin{array}{ll}f_{0}(t) & \text { if } t \in \mathcal{D} \\ \liminf _{s \rightarrow t, s \in \mathcal{D}} f_{0}(s) & \text { if } t \notin \mathcal{D}\end{array} \quad g(t)= \begin{cases}g_{0}(t) & \text { if } t \in \mathcal{D} \\ \lim \sup _{s \rightarrow t, s \in \mathcal{D}} g_{0}(s) & \text { if } t \notin \mathcal{D}\end{cases}\right.$

(indeed $f(t)=\liminf _{s \rightarrow t, s \in \mathcal{D}} f_{0}(s)$, for every $t \in K$ and $g(t)=\lim \sup _{s \rightarrow t, s \in \mathcal{D}} g_{0}(s)$ for every $t \in K$ as well) and finally consider

$$
E:=[f, g]
$$

which is an intersection of closed balls since $f$ and $g$ are finite-real valued ( $f_{0}$ and $g_{0}$ are bounded on $\mathcal{D}$ ). Let us call $E$ the proximal completion of $C$. The reason for this name will soon become clear.

Proposition 3.1. Every $C \in \mathcal{M}$ has a nonempty metric projection in $\gamma(C)$ and

$$
d(C, \gamma(C))=\frac{1}{2}\left(d_{C}-\delta_{C}\right) .
$$

Proof. Consider an arbitrary $C \in \mathcal{M}$ and denote by $f_{C}$ and $g_{C}$ the lower and upper semi-continuous functions, respectively, satisfying $C=\left[f_{C}, g_{C}\right]$. To prove (3.2) we first show that $d(C, \gamma(C)) \geq \frac{1}{2}\left(d_{C}-\delta_{C}\right)$. As a first step, notice that

$$
\mathcal{D} \subset \mathcal{D}_{\delta_{C}}
$$

\footnotetext{
1 This word, a combination of proximity and minimal, first appeared in the first paper by R.R. Phelps [22], written when he was a graduate student. It was suggested to him by a fellow student, Raymond Killgrove.
} 
Indeed, consider $t_{0} \in \mathcal{D}$ and $\varepsilon>0$. Since $\delta_{C}$ is lower semicontinuous, there is a neighborhood $U$ of $t_{0}$ such that $\delta_{C}(t) \geq \delta_{C}\left(t_{0}\right)-\varepsilon$ for every $t \in U$. Since $t_{0} \in$ $\mathcal{D}_{f_{C}} \cap \mathcal{D}_{g_{C}}$, we may assume also that $f_{C}(t) \geq f_{C}\left(t_{0}\right)-\varepsilon$ and $g_{C}(t) \leq g_{C}\left(t_{0}\right)+\varepsilon$ for each $t \in U$. Therefore, if $\psi, \xi \in C$ and $t \in U$, then

$$
|\psi(t)-\xi(t)| \leq g_{C}\left(t_{0}\right)-f_{C}\left(t_{0}\right)+2 \varepsilon=\delta_{C}\left(t_{0}\right)+2 \varepsilon
$$

and hence

$$
\delta_{C}\left(t_{0}\right)-\varepsilon \leq \delta_{C}(t)=\sup _{\psi, \xi \in C}|\psi(t)-\xi(t)| \leq \delta_{C}\left(t_{0}\right)+2 \varepsilon
$$

which proves (3.3). Consider now an arbitrary completion $D=\left[\varphi, \check{\varphi}+d_{C}\right] \in \gamma(C)$ and fix $\varepsilon>0$. It is not difficult to prove that

$$
\delta_{C}=\inf _{t \in \mathcal{D}}\left\{g_{C}(t)-f_{C}(t)\right\}=\inf _{t \in K}\left\{g_{C}(t)-f_{C}(t)\right\}
$$

using, in the case of the first equality, (3.3) and the observation following (2.1); the reasons for the validity of the second equality are the density of $\mathcal{D}$ in $K$ together with the semicontinuity properties of $f_{C}$ and $g_{C}$. Therefore, in virtue of (3.4), we can find $t_{0} \in \mathcal{D}$ such that $g_{C}\left(t_{0}\right)-f_{C}\left(t_{0}\right)<\delta_{C}+\varepsilon$. Since $\varphi$ is lower semicontinuous, $\mathcal{D}_{\varphi}$ is residual, hence we may assume that $t_{0}$ is also a point of continuity for $\varphi$ and therefore also for $\check{\varphi}$. In order to estimate $\operatorname{dist}(C, D)$, we consider two cases. Suppose first that

$$
\varphi\left(t_{0}\right) \leq f_{C}\left(t_{0}\right)-\frac{1}{2}\left(d_{C}-\delta_{C}\right)
$$

Then,

$$
\begin{aligned}
\frac{1}{2}\left(d_{C}-\delta_{C}\right) & \leq f_{C}\left(t_{0}\right)-\varphi\left(t_{0}\right) \\
& =\inf \left\{\xi\left(t_{0}\right): \xi \in C\right\}-\inf \left\{\zeta\left(t_{0}\right): \zeta \in D\right\} \\
& \leq \operatorname{dist}(C, D)
\end{aligned}
$$

If (3.5) does not hold, then $\varphi\left(t_{0}\right)>f_{C}\left(t_{0}\right)-\frac{1}{2}\left(d_{C}-\delta_{C}\right)$ and hence we obtain

$$
\begin{aligned}
\operatorname{dist}(D, C) & \geq \sup \left\{\zeta\left(t_{0}\right): \zeta \in D\right\}-\sup \left\{\xi\left(t_{0}\right): \xi \in C\right\} \\
& =\check{\varphi}\left(t_{0}\right)+d_{C}-g_{C}\left(t_{0}\right) \\
& =\varphi\left(t_{0}\right)+d_{C}-g_{C}\left(t_{0}\right) \\
& >f_{C}\left(t_{0}\right)-g_{C}\left(t_{0}\right)+d_{C}-\frac{1}{2}\left(d_{C}-\delta_{C}\right) \\
& >\frac{1}{2}\left(d_{C}-\delta_{C}\right)-\varepsilon .
\end{aligned}
$$

Having in mind that $\varepsilon$ is arbitrary, we get the desired inequality $d(C, \gamma(C)) \geq \frac{1}{2}\left(d_{C}-\right.$ $\left.\delta_{C}\right)$.

The proof of the proposition will be accomplished by showing that the proximal completion $E$ of $C$ satisfies $E \in \gamma(C)$ and $\operatorname{dist}(C, E)=\frac{1}{2}\left(d_{C}-\delta_{C}\right)$. To show that $E$ is diametrically maximal, we must check that $g(t)-f(t)=\operatorname{diam} E$ for every $t \in \mathcal{D}_{f} \cap \mathcal{D}_{g}$. We know that $g(t)-f(t)=d_{C}$ for every $t \in \mathcal{D}$, and it is important to notice here that

$$
\mathcal{D} \subset \mathcal{D}_{f} \cap \mathcal{D}_{g}
$$


Since $\mathcal{D}$ is a dense set in $K$, it is dense also in $\mathcal{D}_{f} \cap \mathcal{D}_{g}$, hence

$$
g(t)-f(t)=d_{C}
$$

for every $t \in \mathcal{D}_{f} \cap \mathcal{D}_{g}$. Now having in mind that

$$
\operatorname{diam} E=\sup \left\{g(t)-f(t), t \in \mathcal{D}_{f} \cap \mathcal{D}_{g}\right\}
$$

we conclude that $E$ is diametrically maximal and has the same diameter as $C$. Let us prove that $C \subset E$. If $t \in \mathcal{D}$, then

$$
\begin{aligned}
f(t)-f_{C}(t) & =h_{0}(t)-\frac{d_{C}}{2}-f_{C}(t) \\
& =\frac{1}{2}\left(g_{C}(t)+f_{C}(t)\right)-\frac{d_{C}}{2}-f_{C}(t) \\
& =\frac{1}{2}\left(g_{C}(t)-f_{C}(t)-d_{C}\right) \leq 0
\end{aligned}
$$

and, analogously, $g(t)-g_{C}(t) \geq 0$. Since $f$ is lower semicontinuous, $g$ is upper semicontinuous, $\mathcal{D}$ is dense in $K$ and $\mathcal{D} \subset \mathcal{D}_{f} \cap \mathcal{D}_{g}$, both inequalities imply that $\left[f_{C}, g_{C}\right] \subset[f, g]$, as desired.

To finish the proof, we will see that $\operatorname{dist}(C, E) \leq \frac{1}{2}\left(d_{C}-\delta_{C}\right)$. Since $C \subset E$, then

$$
\operatorname{dist}(C, E)=\inf \{\varepsilon>0: E \subset C+\varepsilon B\}
$$

where $B$ is the unit ball. According to Proposition 2.3 in [15], we have

$$
C+\varepsilon B=\left[f_{C}, g_{C}\right]+[-\varepsilon, \varepsilon]=\left[f_{C}-\varepsilon, g_{C}+\varepsilon\right] .
$$

Let $\varepsilon=\frac{1}{2}\left(d_{C}-\delta_{C}\right)$ and consider $t \in \mathcal{D}$. Then

$$
\begin{aligned}
g(t) & =\frac{1}{2}\left(g_{C}(t)+f_{C}(t)\right)+\frac{d_{C}}{2} \\
& \leq \frac{1}{2}\left(g_{C}(t)+f_{C}(t)\right)+\frac{1}{2}\left(g_{C}(t)-f_{C}(t)-\delta_{C}\right)+\frac{d_{C}}{2} \\
& =g_{C}(t)+\frac{1}{2}\left(d_{C}-\delta_{C}\right) .
\end{aligned}
$$

An analogous argument shows that $f(t) \geq f_{C}(t)-\frac{1}{2}\left(d_{C}-\delta_{C}\right)$. Since $\mathcal{D}$ is dense in $K$ and $f_{C}, g_{C}$ are lower and upper semicontinuous, respectively, both inequalities imply that $[f, g] \subset\left[f_{C}-\varepsilon, g_{C}+\varepsilon\right]$ which, together with (3.8), shows that $\operatorname{dist}(C, E) \leq$ $\frac{1}{2}\left(d_{C}-\delta_{C}\right)$ as desired.

4. When is $\gamma(C)$ Chebyshev at $C$ ? The question of whether $\gamma(C)$ is Chebyshev at $C$ is closely related to the range of the mapping $\delta_{C}: K \rightarrow \mathbb{R}$ and actually depends on the cardinality of the set $\left\{\delta_{C}(t): t \in \mathcal{D}_{\delta_{C}}\right\}$, as shown in the next proposition. As a corollary of this result we get that, in $\left(\mathbb{R}^{2},\|\cdot\|_{\infty}\right)$, the family $\gamma(C)$ of completions of an arbitrary intersection of balls $C \subset \mathbb{R}^{2}$ is always Chebyshev at $C$ since, in this case, card $K=2$.

Proposition 4.1. $\gamma(C)$ is Chebyshev at $C$ if and only if there is no point of continuity $t_{0} \in \mathcal{D}_{\delta_{C}}$ satisfying $\delta_{C}<\delta_{C}\left(t_{0}\right)<d_{C}$. More precisely, if $\lambda(t)=\min \left\{d_{C}-\right.$ $\left.\delta_{C}(t), \delta_{C}(t)-\delta_{C}\right\}$, then

$$
\operatorname{diam} \pi(C)=\sup _{t \in \mathcal{D}_{\delta_{C}}} \lambda(t)
$$


Proof. Consider the proximal completion $E=[f, g]$ of $C$ defined in (3.1) and denote $\sigma(t)=\lambda(t) / 2$. We claim that $E^{+}:=[f+\sigma, g+\sigma]$ and $E^{-}:=[f-\sigma, g-\sigma]$ are optimal completions of $C$ satisfying

$$
\operatorname{dist}\left(E^{+}, E^{-}\right)=\sup _{t \in \mathcal{D}_{\delta_{C}}} \lambda(t) .
$$

Let us see first that $E^{+} \in \gamma(C)$ (the proof of $E^{-} \in \gamma(C)$ is analogous). As a consequence of (3.3), we know that $\mathcal{D} \subset \mathcal{D}_{\lambda}=\mathcal{D}_{\sigma}$ and, since $\mathcal{D} \subset \mathcal{D}_{f} \cap \mathcal{D}_{g}$ as well, we get $\mathcal{D} \subset \mathcal{D}_{f+\sigma} \cap \mathcal{D}_{g+\sigma}$. It is clear that, for every $t \in \mathcal{D}_{f} \cap \mathcal{D}_{g}$ and, in particular, for every $t \in \mathcal{D}$, we have

$$
(g+\sigma)(t)-(f+\sigma)(t)=g(t)-f(t)=\operatorname{diam} E
$$

in virtue of (3.6) and (3.7). Since $\mathcal{D}$ is dense in $\mathcal{D}_{f+\sigma} \cap \mathcal{D}_{g+\sigma}$, these equalities also hold for every $t \in \mathcal{D}_{f+\sigma} \cap \mathcal{D}_{g+\sigma}$. Therefore diam $E^{+}=\operatorname{diam} E$ and, according to the characterization given in [18], $E^{+}$is diametrically maximal. We will show now that

$$
C=\left[f_{C}, g_{C}\right] \subset[f+\sigma, g+\sigma]=E^{+}
$$

or, equivalently, that

$$
f(s)+\sigma(s) \leq f_{C}(s) \leq g_{C}(s) \leq g(s)+\sigma(s)
$$

for every $s \in \mathcal{D}$. The proof of $g_{C}(s) \leq g(s)+\sigma(s)$ is straightforward since $g_{C}(s) \leq g(s)$ ( $E^{+}$is a completion of $C$ ) and $\sigma(s) \geq 0$ for every $s \in K$. In the case of the first inequality,

$$
\begin{aligned}
f(s)+\sigma(s) & =h_{0}(s)-\frac{d_{C}}{2}+\sigma(s) \\
& =\frac{1}{2}\left(g_{C}(s)+f_{C}(s)-d_{C}+\lambda(s)\right) \\
& \leq f_{C}(s)+\frac{1}{2}\left(g_{C}(s)-f_{C}(s)-d_{C}+d_{C}-\delta_{C}(s)\right) \\
& =f_{C}(s)
\end{aligned}
$$

where we have used that $\delta_{C}(s)=g_{C}(s)-f_{C}(s)$ when $s \in \mathcal{D}$. Once we know that $E^{+}, E^{-} \in \gamma(C)$, to prove that both sets are indeed optimal completions of $C$ we must check, according to Proposition 3.1, that $\operatorname{dist}\left(C, E^{+}\right)=(1 / 2)\left(d_{C}-\delta_{C}\right)=\operatorname{dist}\left(C, E^{-}\right)$. Indeed, it is enough to show that

$$
\operatorname{dist}\left(C, E^{+}\right) \leq \frac{1}{2}\left(d_{C}-\delta_{C}\right) .
$$

being the corresponding inequality $\operatorname{dist}\left(C, E^{-}\right) \leq(1 / 2)\left(d_{C}-\delta_{C}\right)$ completely analogous. Having again in mind that $C \subset E^{+}$, we just need to show that $[f+\sigma, g+\sigma] \subset$ $\left[f_{C}-\varepsilon, g_{C}+\varepsilon\right]$ with $\varepsilon=(1 / 2)\left(d_{C}-\delta_{C}\right)$ or, equivalently, that

$$
f_{C}(s)-\frac{1}{2}\left(d_{C}-\delta_{C}\right) \leq f(s)+\sigma(s) \leq g(s)+\sigma(s) \leq g_{C}(s)+\frac{1}{2}\left(d_{C}-\delta_{C}\right)
$$

for every $s \in \mathcal{D}$. The first inequality is straightforward since $\sigma \geq 0$ and $\operatorname{dist}(C, E)=$ $\frac{1}{2}\left(d_{C}-\delta_{C}\right)$, while second inequality is trivial. We must prove only the third one which 
is, actually, quite similar to the one proved at the end of the proof of Proposition 3.1:

$$
\begin{aligned}
g(s)+\sigma(s) & =\frac{1}{2}\left(g_{C}(s)+f_{C}(s)+d_{C}+\lambda(s)\right) \\
& \leq \frac{1}{2}\left(g_{C}(s)+f_{C}(s)+d_{C}+\delta_{C}(s)-\delta_{C}\right) \\
& =g_{C}(s)+\frac{1}{2}\left(d_{C}-\delta_{C}\right) .
\end{aligned}
$$

In order to prove formula (4.1) we will calculate $\operatorname{dist}\left(E^{+}, E^{-}\right)$using formula (2.2), since $\mathcal{D} \subset \mathcal{D}_{f+\sigma} \cap \mathcal{D}_{f-\sigma}$ (see the observations following (4.2)), $\mathcal{D} \subset D_{\delta_{C}}$ and $\mathcal{D}$ is dense in $K$ and therefore in every subset of $K$ containing it:

$$
\begin{aligned}
\operatorname{diam} \pi(C) & \geq \operatorname{dist}\left(E^{+}, E^{-}\right) \\
& =\sup _{t \in \mathcal{D}}|(f(t)+\sigma(t))-(f(t)-\sigma(t))| \\
& =\sup _{t \in \mathcal{D}} \lambda(t) \\
& =\sup _{t \in D_{\delta_{C}}} \lambda(t) .
\end{aligned}
$$

To prove the reverse inequality, consider two arbitrary sets $E^{\prime}=\left[f^{\prime}, g^{\prime}\right]$ and $E^{\prime \prime}=$ $\left[f^{\prime \prime}, g^{\prime \prime}\right]$, both in $\pi(C)$. We will show that

$$
\begin{aligned}
\operatorname{dist}\left(E^{\prime}, E^{\prime \prime}\right) & =\sup _{t \in \mathcal{D}_{0}}\left|f^{\prime}(t)-f^{\prime \prime}(t)\right| \\
& \leq \sup _{t \in \mathcal{D}_{0}} \lambda(t) \\
& =\sup _{t \in \mathcal{D}_{\delta_{C}}} \lambda(t)
\end{aligned}
$$

where $\mathcal{D}_{0}$ is the residual set obtained by intersecting the sets of points of continuity of all semicontinuous functions which appear in this proof, namely

$$
\mathcal{D}_{0}:=\mathcal{D} \cap \mathcal{D}_{f^{\prime}} \cap \mathcal{D}_{f^{\prime \prime}} \cap \mathcal{D}_{g^{\prime}} \cap \mathcal{D}_{g^{\prime \prime}}
$$

Equality (4.5) is just again formula (2.2) together with the density of $\mathcal{D}_{0}$ in $\mathcal{D}_{f^{\prime}} \cap \mathcal{D}_{f^{\prime \prime}}$, while (4.7) is due to the denseness of $\mathcal{D}_{0}$ in $\mathcal{D}_{\delta_{C}}$. The proof of (4.6) will be divided in two parts: for every $t \in \mathcal{D}_{0}$ we have (1) $\left|f^{\prime}(t)-f^{\prime \prime}(t)\right| \leq \delta_{C}(t)-\delta_{C}$ and (2) $\left|f^{\prime}(t)-f^{\prime \prime}(t)\right| \leq d_{C}-\delta_{C}(t)$. We begin by showing that, for every $t \in \mathcal{D}_{0}$,

$$
f^{\prime \prime}(t) \geq f(t)-\frac{1}{2}\left(\delta_{C}(t)-\delta_{C}\right)
$$

as a previous step to prove (1). Indeed, Proposition 3.1 and formula (2.2) imply that $f^{\prime \prime}(t) \geq f_{C}(t)-\frac{1}{2}\left(d_{C}-\delta_{C}\right)$ so therefore, having in mind that $f(t)=(1 / 2)\left(g_{C}(t)+\right.$ $\left.f_{C}(t)-d_{C}\right)$, we obtain

$$
\begin{aligned}
f^{\prime \prime}(t) & \geq f_{C}(t)-\frac{1}{2}\left(d_{C}-\delta_{C}\right) \\
& =\frac{1}{2}\left(g_{C}(t)+f_{C}(t)\right)+\frac{1}{2}\left(f_{C}(t)-g_{C}(t)\right)-\frac{1}{2}\left(d_{C}-\delta_{C}\right) \\
& =f(t)-\frac{1}{2}\left(\delta_{C}(t)-\delta_{C}\right) .
\end{aligned}
$$


A similar argument can be used to prove $g^{\prime \prime}(t) \leq g(t)+\frac{1}{2}\left(\delta_{C}(t)-\delta_{C}\right)$. Hence, since $t$ is a point of continuity for $f, g, f^{\prime \prime}$ and $g^{\prime \prime}$, we have $f^{\prime \prime}(t)=g^{\prime \prime}(t)-d_{C}$ and $f(t)=g(t)-d_{C}$. Then

$$
f^{\prime \prime}(t) \leq g(t)+\frac{1}{2}\left(\delta_{C}(t)-\delta_{C}\right)-d_{C}=f(t)+\frac{1}{2}\left(\delta_{C}(t)-\delta_{C}\right) .
$$

Both inequalities (4.8) and (4.9) are also satisfied when we replace $f^{\prime \prime}$ by $f^{\prime}$. As a result,

$$
\left|f^{\prime \prime}(t)-f^{\prime}(t)\right| \leq \delta_{C}(t)-\delta_{C}
$$

and so (1) is proved. To show (2) suppose, on the contrary, that there is $t \in \mathcal{D}_{0}$ such that

$$
\left|f^{\prime \prime}(t)-f^{\prime}(t)\right|>d_{C}-\delta_{C}(t) .
$$

Assume, for instance, that $f^{\prime}(t)>f^{\prime \prime}(t)$, hence $f^{\prime}(t)-f^{\prime \prime}(t)=\left|f^{\prime \prime}(t)-f^{\prime}(t)\right|>$ $d_{C}-\delta_{C}(t)$. Since $C \subset\left[f^{\prime \prime}, g^{\prime \prime}\right]$ and $t \in \mathcal{D}_{0} \subset \mathcal{D}_{g_{C}} \cap \mathcal{D}_{g^{\prime \prime}}$, it is clear that $g^{\prime \prime}(t) \geq g_{C}(t)$; analogously, $f^{\prime}(t) \leq f_{C}(t)$. Therefore

$$
\begin{aligned}
d_{C}=\operatorname{diam} E^{\prime \prime} & =g^{\prime \prime}(t)-f^{\prime \prime}(t) \\
& >g_{C}(t)-f^{\prime}(t)+\left(d_{C}-\delta_{C}(t)\right) \\
& \geq g_{C}(t)-f_{C}(t)+\left(d_{C}-\delta_{C}(t)\right) \\
& =d_{C}
\end{aligned}
$$

which is a contradiction. The proof for the case $f^{\prime}(t)<f^{\prime \prime}(t)$ is completely similar.

5. When is $\mathcal{D} \mathcal{M}$ proximinal in $C \in \mathcal{M}$ ? Once we know that, for an intersection of closed balls $C \in C(K)$, the problem of minimizing dist $(C, E)$ with the constraint $E \in \gamma(C)$ has a solution, the following natural question arises: what if we change the above constraint to the more general one $E \in \mathcal{D} \mathcal{M}$ ? In other words, we want to find the best approximation to $C$ by a diametrically maximal set, with no attention of whether or not it is a completion of $C$. The next proposition shows that this problem also has an optimal solution.

Proposition 5.1. Every $C \in \mathcal{M}$ has a nonempty metric projection in $\mathcal{D} \mathcal{M}$ and

$$
d(C, \mathcal{D} \mathcal{M})=\frac{1}{4}\left(d_{C}-\delta_{C}\right)
$$

Proof. To prove that $d(C, \mathcal{D} \mathcal{M}) \leq \frac{1}{4}\left(d_{C}-\delta_{C}\right)$ we will define a diametrically maximal set $E^{\prime}$ using a similar construction to the one used in the case of the proximal completion $E=[f, g]$ considered in Proposition 3.1. We keep the same notation used there. Define

$$
\begin{aligned}
& f_{0}^{\prime}(t)=h_{0}(t)-\frac{1}{4}\left(d_{C}+\delta_{C}\right) \\
& g_{0}^{\prime}(t)=h_{0}(t)+\frac{1}{4}\left(d_{C}+\delta_{C}\right)
\end{aligned}
$$

for every $t \in \mathcal{D}=\mathcal{D}_{f_{C}} \cap \mathcal{D}_{g_{C}}$ and consider the corresponding lower and upper semicontinuous extensions: $f^{\prime}(t)=\liminf _{s \rightarrow t, s \in \mathcal{D}} f_{0}^{\prime}(s)$ and $g^{\prime}(t)=\limsup _{s \rightarrow t, s \in \mathcal{D}} g_{0}^{\prime}(s)$. It 
can be proved, in a similar manner as done with $E$, that $E^{\prime}=\left[f^{\prime}, g^{\prime}\right]$ is a diametrically maximal set, with diameter $\frac{1}{2}\left(d_{C}+\delta_{C}\right)$. We claim that $\operatorname{dist}\left(C, E^{\prime}\right)=\frac{1}{4}\left(d_{C}-\delta_{C}\right)$. To prove this fact, we will check first that

$$
E^{\prime}+\frac{1}{4}\left(d_{C}-\delta_{C}\right) B=E
$$

hence $C \subset E^{\prime}+\frac{1}{4}\left(d_{C}-\delta_{C}\right) B$. Indeed, according to Proposition 2.3 in [15],

$$
\begin{aligned}
E^{\prime}+\frac{1}{4}\left(d_{C}-\delta_{C}\right) B & =\left[f^{\prime}, g^{\prime}\right]+\frac{1}{4}\left(d_{C}-\delta_{C}\right) B \\
& =\left[f^{\prime}-\frac{1}{4}\left(d_{C}-\delta_{C}\right), g^{\prime}+\frac{1}{4}\left(d_{C}-\delta_{C}\right)\right]
\end{aligned}
$$

and moreover, if $t \in \mathcal{D}$,

$$
\begin{aligned}
f^{\prime}(t)-\frac{1}{4}\left(d_{C}-\delta_{C}\right) & =h_{0}(t)-\frac{1}{4}\left(d_{C}+\delta_{C}\right)-\frac{1}{4}\left(d_{C}-\delta_{C}\right) \\
& =h_{0}(t)-\frac{1}{2} d_{C}=f_{0}(t)=f(t)
\end{aligned}
$$

and, analogously, $g^{\prime}(t)+\frac{1}{4}\left(d_{C}-\delta_{C}\right)=g(t)$. Since $f, f^{\prime}$ are lower-semicontinuous, $g, g^{\prime}$ are upper-semicontinuous and $\mathcal{D}$ is dense in $K$, the above equalities proves (5.1). Since $E$ is a completion of $C$, we obtain that $C \subset E=E^{\prime}+\frac{1}{4}\left(d_{C}-\delta_{C}\right) B$. The inclusion $E^{\prime} \subset C+\frac{1}{4}\left(d_{C}-\delta_{C}\right) B$ follows from the following estimates: if $t \in \mathcal{D}$, then

$$
\begin{aligned}
f^{\prime}(t)=h_{0}(t)-\frac{1}{4}\left(d_{C}+\delta_{C}\right) & =f_{C}(t)+\frac{1}{2} \delta_{C}(t)-\frac{1}{4}\left(d_{C}-\delta_{C}\right) \\
& \geq f_{C}(t)-\frac{1}{4}\left(d_{C}-\delta_{C}\right)
\end{aligned}
$$

and, analogously, $g^{\prime}(t) \leq g_{C}(t)+\frac{1}{4}\left(d_{C}-\delta_{C}\right)$. These inequalities together with the density of $\mathcal{D}$ and the semicontinuity properties of $f_{C}$ and $g_{C}$ give the desired inclusion.

In order to prove that $d(C, \mathcal{D} \mathcal{M}) \geq \frac{1}{4}\left(d_{C}-\delta_{C}\right)$ we consider an arbitrary $F \in \mathcal{D} \mathcal{M}$ and we will show that $\operatorname{dist}(C, F) \geq \frac{1}{4}\left(d_{C}-\delta_{C}\right)$. Denote $F=\left[f_{F}, g_{F}\right]$, where $f_{F}$ and $g_{F}$ are lower and upper semicontinuous, respectively, and let $\mathcal{D}_{F}=\mathcal{D}_{f_{F}} \cap \mathcal{D}_{g_{F}}$. The proof of the above inequality will be divided in two cases, according to the size of the diameter of $F$.

Case 1: diam $F<\frac{1}{2}\left(d_{C}+\delta_{C}\right)$. If there is $t \in \mathcal{D} \cap \mathcal{D}_{F}$ satisfying $\left|g_{C}(t)-g_{F}(t)\right|>$ $\frac{1}{4}\left(d_{C}-\delta_{C}\right)$ we are done, since $\operatorname{dist}(C, F) \geq \sup _{t \in \mathcal{D} \cap \mathcal{D}_{F}}\left|g_{C}(t)-g_{F}(t)\right|$. Otherwise, assume that $\left|g_{C}(t)-g_{F}(t)\right| \leq \frac{1}{4}\left(d_{C}-\delta_{C}\right)$ for every $t \in \mathcal{D} \cap \mathcal{D}_{F}$. Given $\varepsilon>0$, we can choose $t_{0} \in \mathcal{D} \cap \mathcal{D}_{F}$ such that $g_{C}\left(t_{0}\right)-f_{C}\left(t_{0}\right) \geq d_{C}-\varepsilon$. Then

$$
\begin{aligned}
\operatorname{dist}(C, F) \geq f_{F}\left(t_{0}\right)-f_{C}\left(t_{0}\right) & =g_{F}\left(t_{0}\right)-\operatorname{diam} F-f_{C}\left(t_{0}\right) \\
& \geq g_{C}\left(t_{0}\right)-\frac{1}{4}\left(d_{C}-\delta_{C}\right)-\operatorname{diam} F-f_{C}\left(t_{0}\right) \\
& >d_{C}-\varepsilon-\frac{1}{4}\left(d_{C}-\delta_{C}\right)-\frac{1}{2}\left(d_{C}+\delta_{C}\right) \\
& \geq \frac{1}{4}\left(d_{C}-\delta_{C}\right)-\varepsilon .
\end{aligned}
$$

Case 2: diam $F \geq \frac{1}{2}\left(d_{C}+\delta_{C}\right)$. As in the previous case, and for the same reason, we may assume that $\left|g_{C}(t)-g_{F}(t)\right| \leq \frac{1}{4}\left(d_{C}-\delta_{C}\right)$ for every $t \in \mathcal{D} \cap \mathcal{D}_{F}$. Given $\varepsilon>0$, 
we can choose $s_{0} \in \mathcal{D} \cap \mathcal{D}_{F}$ such that $g_{C}\left(s_{0}\right)-f_{C}\left(s_{0}\right) \leq \delta_{C}+\varepsilon$. Then

$$
\begin{aligned}
\operatorname{dist}(C, F) \geq f_{C}\left(s_{0}\right)-f_{F}\left(s_{0}\right) & \geq g_{C}\left(s_{0}\right)-\delta_{C}-\varepsilon-f_{F}\left(s_{0}\right) \\
& \geq g_{F}\left(s_{0}\right)-\frac{1}{4}\left(d_{C}-\delta_{C}\right)-\delta_{C}-\varepsilon-f_{F}\left(s_{0}\right) \\
& =\operatorname{diam} F-\frac{1}{4}\left(d_{C}-\delta_{C}\right)-\delta_{C}-\varepsilon \\
& \geq \frac{1}{2}\left(d_{C}+\delta_{C}\right)-\frac{1}{4}\left(d_{C}-\delta_{C}\right)-\delta_{C}-\varepsilon \\
& =\frac{1}{4}\left(d_{C}-\delta_{C}\right)-\varepsilon
\end{aligned}
$$

口

6. Final remarks. The diameter of the metric projection of $C$ onto the family $\mathcal{D M}$ can be calculated by using similar arguments than in the proof of Proposition 4.1 and satisfies the same formula (4.1).

Problem $\mathrm{P}$ can be formulated also in a more general form: how far from being diametrically maximal is a closed convex and bounded set $C$ ? The difficulties here are due to the fact that arbitrary convex sets in $C(K)$, in contrast with those which are intersections of balls, may have no suitable representation. One can be tempted to think that, considering $\beta(C)$ (the intersection of all closed balls containing $C$ ), the solutions to Problem 1 for $\beta(C)$ will do the job, but this is not so. Consider, for instance, $C(K)=\left(\mathbb{R}^{2},\|\cdot\|_{\infty}\right)$. Define

$$
C=\operatorname{conv}\{(-2,4),(2,4),(2,-4)\}
$$

for which $\beta(C)=\operatorname{conv}\{C \cup\{(-2,-4)\}\}$. The solution for Problem 1 with the initial data $\beta(C)$ is $\pi(\beta(C))=4 B$, where $B$ is the unit ball. It is not difficult to check that $\operatorname{dist}(C, 4 B)=4$. However, if we consider $D=(1,0)+4 B$, which is also a completion of $\beta(C)$, then $\operatorname{dist}(C, D)<4$. Of course, Problem 1 with initial data $C$ has in this case a solution because, due to the famous Blaschke selection theorem [27], the family $\gamma(C)$ is compact and this also applies to every normed finite dimensional space and fails to be true in infinite dimensional normed spaces.

The author would like to acknowledge the valuable suggestions and comments made by R.R. Phelps on the subject of this note. He also thanks the referee for careful reading, useful comments and improvements in exposition.

\section{REFERENCES}

[1] I. BÁRÁNY, Random polytopes, convex bodies, and approximation, Stochastic geometry, pp. 77118, Lecture Notes in Math., 1892, Springer, Berlin, 2007.

[2] M. Baronti and P. L. PAPini, Diameters, centers and diametrically maximal sets, Rend. Circolo Mat. Palermo Suppl. (II), 38 (1995), pp. 11-24.

[3] E. Behrends And P. Harmand, Banach spaces which are proper M-ideals, Studia Math., 81 (1985), pp. 159-169.

[4] V. G. Boltyanski, H. Martini and P. S. Soltan, Excursions into Combinatorial Geometry, Springer, Berlin 1997.

[5] P. Bandyopadhyay, Y. Li, B.-L. Lin, and D. Narayana, Proximinality in Banach spaces, J. Math. Anal. Appl., 341:1 (2008), pp. 309-317.

[6] Y. Benyamini, R. Espínola, And G. LÓPez, Nonexpansive selections of metric projections in spaces of continuous functions, J. Approx. Theory, 137:2 (2005), pp. 187-200.

[7] G. D. Chakerian and H. Groemer, Convex bodies of constant width, in "Convexity and its Applications", P. Gruber and J. Wills, Eds, Birkhäuser, (1983), pp. 49-96. 
[8] F. Deutsch, Best approximation in Inner Product spaces, CMS Books in Mathematics/Ouvrages de Mathématiques de la SMC, 7. Springer-Verlag, New York, 2001.

[9] H. G. EgGLeston, Sets of constant width in finite dimensional Banach spaces, Israel J. Math., 3 (1965), pp. 163-172.

[10] V. Indumathi and S. Lalithambigai, A new proof of proximinality for $M$-ideals, Proc. Amer. Math. Soc., 135:4 (2007), pp. 1159-1162.

[11] K.-S. LaU, On a sufficient condition for proximity, Trans. Amer. Math. Soc., 251 (1979), pp. 343-356.

[12] W. A. Kirk, S. Reich and P. Veeramani, Proximinal retracts and best proximity pair theorems, Numer. Funct. Anal. Optim., 24:7-8 (2003), pp. 851-862.

[13] P. Koszmider, A space $C(K)$ where all nontrivial complemented subspaces have big densities, Studia Math., 168:2 (2005), pp. 109-127.

[14] E. Meissner, Über Punktmengen konstanter Breite, Vierteljahresschr. naturforsch. Ges Zürich, 56 (1911), pp. 42-50. Jbuch. 42, p. 91.

[15] J. P. Moreno, Semicontinuous functions and convex sets in $C(K)$ spaces, J. Austral. Math. Soc., 82 (2007), pp. 111-121.

[16] J. P. Moreno, Porosity and diametrically maximal sets in $C(K)$, Monatsh. Math., 152 (2007), pp. 255-263.

[17] J. P. Moreno, Convex values and Lipschitz behavior of the complete hull mapping, Trans. Amer. Math. Soc, 362 (2010), pp. 3377-3389.

[18] J. P. Moreno, P. L. Papini and R. R. Phelps, Diametrically maximal and constant width sets in Banach spaces, Canad. J. Math., 58:4 (2006), pp. 820-842.

[19] J. P. Moreno, P. L. Papini and R. R. Phelps, New families of convex sets related to diametral maximality, J. Convex. Anal., 13 (2006), pp. 823-837.

[20] D. Narayana And T. S. S. R. K. RaO, Transitivity of proximinality and norm attaining functionals, Colloq. Math., 104:1 (2006), pp. 1-19.

[21] D. Pallaschke and S. Rolewicz, Foundations of mathematical optimization. Convex analysis without linearity, Mathematics and its Applications, 388. Kluwer Academic Publishers Group, Dordrecht, 1997.

[22] R. R. Phelps, Convex sets and nearest points, Proc. Amer. Math. Soc., 8 (1957), pp. 790-797.

[23] R. Paya and A. Rodríguez-Palacios, Banach spaces which are semi-L-summands in their biduals, Math. Ann., 289 (1991), pp. 529-542.

[24] E. S. Polovinkin, Convex bodies of constant width, Doklady Mathematics, 3 (2004), pp. 313315. Traslated in English (pp. 560-562 for the translation).

[25] S. Reich and A. Zaslavski, Porous sets and generalized best approximation problems, Nonlinear Anal. Forum, 9:2 (2004), pp. 135-152.

[26] A. Rodríguez-Palacios, Properly semi-L-embedded complex spaces, Studia Math., 106 (1993), pp. 197-202.

[27] R. Schneider, Convex bodies: The Brunn-Minkowski theory, Encyclopedia of Mathematics and its Applications, 44. Cambridge University Press, Cambridge 1993.

[28] I. Singer, The theory of best approximation and functional analysis. Conference Board of the Mathematical Sciences Regional Conference Series in Applied Mathematics, No. 13. Society for Industrial and Applied Mathematics, Philadelphia, Pa., 1974.

[29] A. J. Zaslavski, Generic existence of solutions in vector optimization on metric spaces, J. Optim. Theory Appl., 136:1 (2008), pp. 139-153. 
J. P. MORENO 